\title{
Noncontrast magnetic resonance imaging versus ultrasonography for hepatocellular carcinoma surveillance (MIRACLE-HCC): study protocol for a prospective randomized trial
}

\author{
Chansik An ${ }^{1}$, Do Young Kim², Jin-Young Choi ${ }^{1}$, Kwang Hyub Han ${ }^{2}$, Yun Ho Roh ${ }^{3}$ and Myeong-Jin Kim ${ }^{1 *}$ (D)
}

\begin{abstract}
Background: Biannual ultrasound (US) — with or without alpha-fetoprotein (AFP)—is recommended by current guidelines for the surveillance of hepatocellular carcinoma (HCC). However, the inadequate sensitivity of US has been a concern. Magnetic resonance imaging (MRI) is known to have high sensitivity in detecting hepatic malignancies, even without contrast enhancement. The purpose of our study is to compare US with noncontrast (unenhanced) MRI for HCC surveillance of high-risk patients.
\end{abstract}

Methods/design: MIRACLE-HCC (usefulness of noncontrast Magnetlc Resonance imAging versus nonContrast ultrasonography for surveiLlancE of HepatoCellular Carcinoma) is a prospective, single-center, nonblinded, balancedrandomized, parallel-group study. This study was approved by our institutional review board, and informed consent will be obtained from all participating patients. All patients with compensated liver cirrhosis will undergo noncontrast US or MRI, with serum AFP testing every 6 months. If a suspicious lesion is newly detected, or if the serum AFP level is elevated in an increasing trend for two consecutive tests, dynamic contrast-enhanced imaging will be performed to confirm the diagnosis. The primary endpoints are detection rates of very early or early stage HCC, stage distribution at the initial diagnosis, and false positive referral rates, which will be compared using Fisher's exact or chi-square tests. The study will include 416 patients in a tertiary academic medical center in South Korea.

Discussion: MIRACLE-HCC is the first prospective randomized trial to compare the effectiveness of noncontrast MRI and noncontrast US in the surveillance of HCC in at-risk patients. The results of this trial will show whether noncontrast MRI surveillance is superior to noncontrast US surveillance in the early detection of HCC. The trial will also determine whether there are fewer false referrals with noncontrast MRI than with noncontrast US and, eventually, whether there is improvement in the overall survival of HCC patients.

Trial registration: The date of trial registration (ClincalTrials.gov: NCT02514434) for this study is July 23, 2015. Enrollment of participants was finished in November 2017. No authors have relationships, conditions, or circumstances that present potential conflicts of interest.

Keywords: Hepatocellular carcinoma, Surveillance, Magnetic resonance imaging, Ultrasonography

\footnotetext{
*Correspondence: kimnex@yuhs.ac

${ }^{1}$ Department of Radiology, Research Institute of Radiological Science, Yonsei

University College of Medicine, 50 Yonsei-ro, Seodaemun-gu, Seoul 03722,

South Korea

Full list of author information is available at the end of the article
}

(C) The Author(s). 2018 Open Access This article is distributed under the terms of the Creative Commons Attribution 4.0 International License (http://creativecommons.org/licenses/by/4.0/), which permits unrestricted use, distribution, and reproduction in any medium, provided you give appropriate credit to the original author(s) and the source, provide a link to the Creative Commons license, and indicate if changes were made. The Creative Commons Public Domain Dedication waiver (http://creativecommons.org/publicdomain/zero/1.0/) applies to the data made available in this article, unless otherwise stated. 


\section{Background}

Periodic surveillance of patients at high risk of developing hepatocellular carcinoma (HCC) is known to improve early tumor detection and overall survival [1-4]. Biannual noncontrast ultrasound (US) - with or without alpha-fetoprotein (AFP) - is recommended as a surveillance imaging modality by the current guidelines, mainly because of wide accessibility and low cost [5-8]. However, the inadequate sensitivity of US has been a concern [9], with a meta-analysis reporting a pooled sensitivity of $63 \%$ for detecting early stage HCC [10]. Moreover, the reported sensitivities of US vary widely-from 32 to $92 \%$-in clinical practice $[11,12]$, demonstrating that US results can be greatly affected by several factors, including imaging protocols, equipment, patient characteristics, and operator experience [13-15].

Given the limited effectiveness of noncontrast US, other imaging modalities, such as contrast-enhanced US, contrast-enhanced computed tomography (CT), and contrast-enhanced magnetic resonance imaging (MRI), have been suggested for use in HCC surveillance [16-21]. However, contrast-enhanced US still has the limitation of being greatly influenced by patient characteristics (i.e., poor echogenic window or liver macro-nodularity) and operator experience, and CT may not be a desirable surveillance modality due to the potential risk of radiation exposure [22]. Although contrast-enhanced MRI is superior to noncontrast (unenhanced) US in detecting small tumors in patients with chronic liver disease [23], its use in HCC surveillance may be hampered by limited accessibility and high cost, as well as the issue of gadolinium-based contrast agent accumulation in human organs [24].

Even without contrast enhancement, MRI is likely to show higher sensitivity than US in detecting hepatic malignancies [25]. Furthermore, noncontrast MRI does not include dynamic sequences using contrast agents, thereby reducing the cost and scanning time, and avoiding the accumulation of gadolinium-based contrast media. We believe that these advantages could make noncontrast MRI a potential alternative to US for HCC surveillance. Therefore, we set up a prospective randomized trial to compare noncontrast US with noncontrast MRI in HCC surveillance of high-risk patients.

\section{Methods/design}

\section{Study design and setting}

The current trial (usefulness of noncontrast MagnetIc Resonance imAging versus nonContrast ultrasonography for surveiLlancE of HepatoCellular Carcinoma [MIRACLE-HCC]) is a prospective, single-center, nonblinded, balanced-randomized, parallel-group study. This trial is registered with ClinicalTrials.gov (identifier: NCT02514434), and the date of registration is July 23, 2015.
This study has been approved by our institutional review board (IRB) and will be conducted in accordance with the ethical principles stated in the Declaration of Helsinki and Good Clinical Practice guidelines. Any modifications to the protocol will require a formal amendment to the protocol and will be reviewed by the IRB. These modifications will be implemented only after they are approved by the IRB and notified to all participating investigators and patients. From August 2015, we recruited patients who started or had been under surveillance for $\mathrm{HCC}$ at the liver cancer center of Severance Hospital, a 2260-bed academic tertiary referral hospital in Seoul, South Korea. Before inclusion in the study, informed written consent has been obtained from each patient. The last patient was enrolled on November 29, 2017, and follow-up to see primary and secondary endpoints is still ongoing.

In South Korea, whose total population is approximately 51 million, the crude incidence rate of liver cancer in 2013 was 32 per 100,000 [26], and 62.7\% of $\mathrm{HCC}$ cases are attributable to chronic hepatitis B virus infection [27]. In Korea, liver cancer is the second-most common cause of death from cancer and was estimated to be responsible for approximately 11,560 deaths in 2014 and ranked first in terms of the total economic burden during 2001-2010 [28, 29].

\section{Eligibility criteria}

Eligible participants are adults aged 20-70 without history of liver cancer who are at risk of developing primary hepatic carcinoma (hepatitis $\mathrm{B}$ surface antigen positive [HBsAg+] or anti-hepatitis $\mathrm{C}$ virus positive [anti-HCV+] with cirrhosis, or cirrhosis from any causes), who have preserved liver function (Child-Pugh class A), and for whom the absence of a liver tumor has been confirmed clinically (no symptoms and no elevated tumor marker) and radiologically (on US, CT, or MRI) at the time of screening. Exclusion criteria are diagnosis of malignancy in the last 5 years, possible pregnancy, and severe cardiovascular, respiratory, renal, or infectious disease. The presence of cirrhosis will be determined based on the histologic or radiologic findings, including liver surface nodularity, margin blunting, segmental hypertrophy/atrophy, and findings secondary to portal hypertension (i.e., splenomegaly, esophageal or gastric varices, ascites, or reverse portal vein flow).

\section{Participant flow}

A trained research nurse will introduce the trial to potentially eligible patients, who will be provided with detailed information about the trial. They will then be able to have an informed discussion with participating physicians, who will obtain written consent if the patients agree to participate in the trial. Enrolled 
participants will be randomly assigned to either abdominal US or noncontrast MRI in addition to serum AFP testing for HCC surveillance (Fig. 1). They will be evaluated by at least 10 rounds of surveillance tests at 6-month intervals (with a variation of \pm 1 month allowed). The first surveillance test will be performed 6 months after the most recent imaging study showing no evidence of hepatic malignancy. If a new lesion detected during US or MRI surveillance is suspected to be malignant according to the predefined criteria (see Imaging evaluation section) or if the serum AFP level is elevated in an increasing trend for two consecutive tests, dynamic contrast-enhanced CT will be performed $[6,30]$. The reference value for AFP is less than $9 \mathrm{ng} / \mathrm{mL}$ at our institution. Additional dynamic liver MRI may be performed at the discretion of clinicians. If the surveillance test result is determined to be a false positive by subsequent tests, the patient will return for the next scheduled surveillance test. If a patient is confirmed as having a hepatic malignancy, the patient will receive standard treatment according to the guidelines [5-8]. We will continue follow-up of treated patients for at least 5 years to obtain and compare the overall survival rates.

Unscheduled examinations may be performed under various circumstances. For example, a participant may visit the hospital and undergo dynamic contrast-enhanced CT between surveillance tests because of symptoms such as abdominal pain or hematemesis. We will

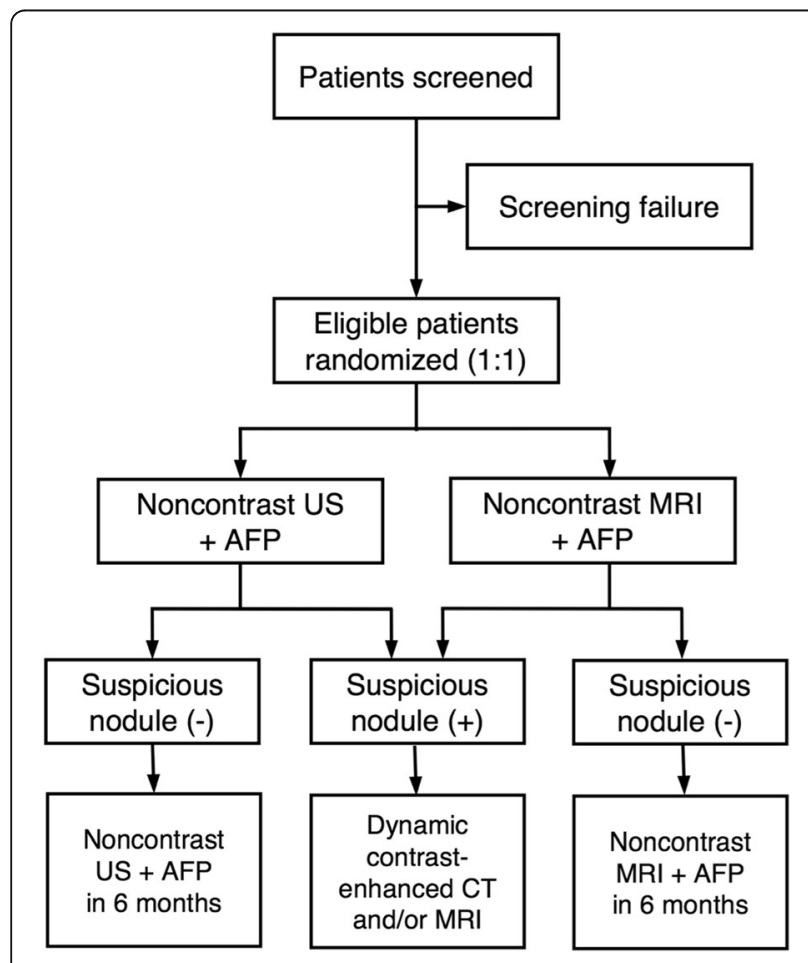

Fig. 1 Participant flow record and report all such cases of unscheduled visits and examinations.

A patient will be considered a dropout, if that participant 1) withdraws consent, 2) receives liver transplantation without diagnosis of liver cancer, 3) is diagnosed as having malignancies other than liver cancer, or 4) does not take two or more consecutive surveillance tests during the trial. To minimize dropout, we will periodically contact participants to remind them of their next scheduled surveillance and to encourage participation. We will also make efforts to obtain relevant information from patients lost to follow-up; for example, if a patient is transferred to a different hospital after hepatic malignancy is diagnosed through this trial, we will contact the patient and ask for information on the results of follow-up treatment or pathological diagnosis.

\section{Ultrasonography}

Abdominal noncontrast US will be performed using commercially available machines (Pro-Sound Alpha10 or Pro-Sound F75, Hitachi Aloka Medical, Tokyo, Japan; ACUSON S2000, Siemens Medical Solutions, Mountain View, CA, USA; iU22, Philips Medical Systems, Best, The Netherlands) with $5-\mathrm{MHz}$ curved-array transducers. Instead of designating specific investigators to perform surveillance US for this trial, we will perform surveillance US as in our current clinical practice. At our institution, surveillance US is performed by well-trained hepatology fellows. They are trained before they start practicing abdominal US, and during the first month of practice, they perform US under the guidance and supervision of faculty members. In performing US, operators follow the guidelines of the Korean Society of Ultrasound in Medicine [31].

\section{MRI}

MRI will be performed using four 3.0-T systems (MAGNETOM Trio Tim, Siemens Healthcare, Erlangen, Germany; Prisma Fit, Siemens Healthcare; Discovery MR 750, GE Healthcare, Milwaukee, WI, USA; Ingenia CX, Philips Healthcare, Best, the Netherlands). All images will be obtained in the transverse plane with a field of view of $44 \mathrm{~cm} \times 33 \mathrm{~cm}$ or $40 \mathrm{~cm} \times 30 \mathrm{~cm}$, according to the patient's body size. After obtaining the localizer images (scanning time: $\sim 20 \mathrm{~s}$ ), T1-weighted images will be obtained by dual-echo T1-weighted gradient-recalled echo (GRE) ( $20 \mathrm{~s})$ and three-dimensional volume interpolated GRE $(\sim 20 \mathrm{~s})$ sequences. T2-weighted images will be acquired by single-shot fat-suppressed navigator-triggered fast or turbo spin echo sequence (3-4 min, depending on the regularity of respiration and patient compliance) and by single-shot breathhold fast or turbo spin echo sequence with a long echo time of 140-150 msec (scanning time: $\sim 20 \mathrm{~s}$ ). We 
will also obtain free breathing or respiratory gated diffusion-weighted images with b-values of 50, 400, and $800 \mathrm{~s} / \mathrm{mm}^{2}(2-4 \mathrm{~min})$, respectively. The apparent diffusion coefficient will be automatically calculated and displayed as a corresponding map. The total scanning time will be approximately $7-10 \mathrm{~min}$, depending on the MRI machine, and the regularity of patients' respiration and their compliance. Details regarding MRI parameters are presented in Table 1.

\section{Imaging evaluation}

Surveillance US findings will be interpreted and reported by the well-trained hepatology fellows. During US, a suspicious nodule on US is defined as a newly appearing nodule $>1 \mathrm{~cm}$. Diffuse infiltrative lesions with or without suspected tumor in the vein are also considered suspicious for HCC. Simple cysts or lesions that have been previously diagnosed as benign lesions, such as hemangiomas, and show no significant interval change are not considered suspicious, irrespective of the size.

Surveillance noncontrast MRI will be evaluated by one of three board-certified abdominal radiologists (MJK, JYC, and CA). At noncontrast MRI, a suspicious nodule is defined as a newly appearing nodule $>1 \mathrm{~cm}$ that shows at least one of the following imaging features: T1 hypointensity, T2 hyperintensity, diffusion restriction, nodule-in-nodule pattern (mosaic appearance), iron sparing, heterogeneous fatty changes, blood products, or definite tumor diameter increase. Diffuse infiltrative lesions with or without a suspected tumor in the vein are also considered suspicious. Simple cysts, hemangiomas, and lesions that have been previously diagnosed as benign lesions are not considered suspicious, irrespective of the size.

Diagnostic dynamic contrast-enhanced CT or MRI will be evaluated and reported using the Liver Imaging Reporting and Data System (LI-RADS) [30], by one of the three board-certified abdominal radiologists (MJK, JYC, and CA). LI-RADS version 2014 was used at our institution until June 2017; after that date, we have been using LI-RADS version 2017.

\section{Reference standards}

The final diagnosis will preferably be determined based on histopathologic findings through surgery (when appropriate, according to the practice guidelines) or biopsy. When histopathologic diagnosis is not possible (i.e., if a patient refuses or cannot tolerate an invasive procedure) or when invasive biopsy is deemed to do

Table 1 Parameters of surveillance noncontrast MRI

\begin{tabular}{|c|c|c|c|c|c|c|c|}
\hline Sequence & Scanner $^{a}$ & Matrix Size & $\mathrm{ST}(\mathrm{mm})$ & Gap (mm) & TR (msec) & TE (msec) & $\overline{F A}\left({ }^{\circ}\right)$ \\
\hline \multirow[t]{4}{*}{ Dual-echo T1-WI GRE } & Magnetom (2D) & $256 \times 192$ & 6 & 1.2 & 150 & $1.23 / 2.46$ & 65 \\
\hline & Ingenia (3D) & $278 \times 256$ & 2 & 0 & 3.2 & $1.15 / 2.30$ & 10 \\
\hline & Discovery (3D) & $320 \times 256$ & 2 & 0 & 3.9 & $1.12 / 2.35$ & 12 \\
\hline & Prisma (3D) & $320 \times 256$ & 2 & 0 & 9 & $1.34 / 2.73$ & 9 \\
\hline \multirow[t]{4}{*}{ T1-WI 3D GRE } & Magnetom & $256 \times 192$ & 2 & 0 & 2.54 & 0.95 & 13 \\
\hline & Ingenia & $256 \times 282$ & 2 & 0 & 3.1 & 1.42 & 10 \\
\hline & Discovery & $320 \times 288$ & 2 & 0 & 4.2 & 1.9 & 12 \\
\hline & Prisma & $320 \times 256$ & 2 & 0 & 2.68 & 1.08 & 9 \\
\hline \multirow[t]{4}{*}{ T2-WI with fat saturation } & Magnetom & $256 \times 192$ & 4 & 1 & 466 & 96 & 150 \\
\hline & Ingenia & $320 \times 212$ & 4 & 1 & 758 & 80 & 90 \\
\hline & Discovery & $320 \times 256$ & 4 & 1 & 2800 & 80 & 90 \\
\hline & Prisma & $320 \times 182$ & 4 & 1 & 620 & 105 & 107 \\
\hline \multirow[t]{4}{*}{ T2-WI with long TE } & Magnetom & $320 \times 168$ & 4 & 1 & 450 & 148 & 150 \\
\hline & Ingenia & $320 \times 186$ & 4 & 1 & 522 & 150 & 90 \\
\hline & Discovery & $320 \times 224$ & 4 & 1 & 840 & 150 & 90 \\
\hline & Prisma & $320 \times 208$ & 4 & 1 & 600 & 153 & 98 \\
\hline \multirow[t]{4}{*}{ DWI } & Magnetom & $128 \times 96$ & 6 & 1 & 5200 & 67 & 90 \\
\hline & Ingenia & $128 \times 128$ & 5 & 1 & 4848 & 55 & 90 \\
\hline & Discovery & $128 \times 80$ & 5 & 1 & 4800 & 51 & 90 \\
\hline & Prisma & $140 \times 112$ & 5 & 1 & 5500 & 63 & 90 \\
\hline
\end{tabular}

${ }^{a}$ Full scanner names are Magnetom Trio Tim (Siemens), Ingenia CX (Philips), and Discovery MR750 (GE), and Prisma Fit (Siemens) 2D Two-dimensional, 3D Three-dimensional, ST Slice thickness, TR Repetition time, TE Echo time, FA Flip angle, GRE Gradient-recalled echo, DWI Diffusion-weighted imaging 
more harm than good (i.e., a patient with radiologically definite $\mathrm{HCC}$ at an advanced stage in whom curative treatment is not indicated), radiologic diagnosis will be applied. For radiologic diagnosis, lesions categorized as LR-5, LR-5 V (LR-TIV in LI-RADS version 2017), or LR-M according to the LI-RADS diagnostic algorithm will be diagnosed as hepatic malignancies [30].

\section{Outcomes}

The primary endpoints are detection rates of very early or early stage HCC, stage distribution at the initial diagnosis, and false positive referral rates. The detection rate is defined as the number of patients whose liver cancer is detected using a given surveillance modality divided by the total number of patients under surveillance with the modality, expressed as a percentage. Very early stage cancer is defined as a single tumor $<2 \mathrm{~cm}$, and early stage cancer is defined as a single tumor $<5 \mathrm{~cm}$ or up to three tumors $<3 \mathrm{~cm}[7,8]$. Surveillance is considered a failure when patients are diagnosed at late (i.e., intermediate or advanced) stages. The false positive rate is defined as the number of positive tests that are eventually confirmed as negative on subsequent dynamic imaging and/or pathologic examination divided by the total number of tests in a specific surveillance modality, expressed as a percentage. The secondary endpoints are 5- and 10-year overall survival rates. We will report these long-term outcomes in separate papers.

\section{Sample size}

Based on the past prevalence of HCC in at-risk patients undergoing surveillance in our institution, we anticipate that the prevalence of HCC in our study cohort will be approximately $18 \%$ over a study period of 5 years. The diagnostic sensitivities of noncontrast US and noncontrast MRI are expected to be $60 \%$ and $90 \%$, respectively $[10,25]$. Based on these expected prevalence and sensitivities, and an anticipated dropout rate of $15 \%$, we calculated that we would need a sample size of 416 patients (208 for each group) to yield $80 \%$ power (1 minus the probability of a type II error) to detect a significant difference in detection rate between the noncontrast US and noncontrast MRI arms, with a two-sided type I error of $5 \%$ [32].

\section{Randomization and blinding}

A research nurse will assign participants into either the noncontrast US group or the noncontrast MRI surveillance group according to a computer-generated randomization list. The randomization sequence will be created using SAS 9.2 (SAS Institute, Inc., Cary, NC, USA) statistical software by an investigator with no clinical involvement in the trial, with a 1:1 allocation using a random block size of 4 . Only the research nurse will have knowledge of the randomization sequence. However, once investigators obtain a participant's consent and the research nurse assigns him or her to the noncontrast US or noncontrast MRI group, the participant and investigators will inevitably be aware of the surveillance assignment at the time of the initial surveillance examination. Therefore, blinding of participants and investigators is not possible for this trial.

\section{Data management}

We will use the Electronic Case Reporting System with Accuracy, Safety, and Efficacy (e-CASE) - a web-based case report form (https://ecase.yuhs.ac) - for data management. The research nurse will enter data into the e-CASE, and a trained data manager will periodically check the accuracy and completeness of the entered data. The data will be kept in the archive of the data center, which has built-in security features preventing unauthorized access. Only investigators, a research nurse, and an independent monitor who will perform data monitoring and auditing will have access to the full dataset. Personal information about participants will be coded and depersonalized.

\section{Statistical analysis}

Both intention-to-treat (considering all patients as randomized irrespective of the surveillance test they actually receive) and per-protocol analyses will be performed. The detection rate, stage distribution, and false positive rates will be compared between the noncontrast US and noncontrast MRI arms using the Fisher's exact or chi-square test. To compare the variables between the two groups, we will use the independent t-test or Mann-Whitney $U$ test for continuous variables and the chi-square or Fisher exact test for categorical variables. If necessary, we will perform logistic regression analysis to determine the independent associations between variables. Survival will be compared using the Kaplan-Meier methods with log-rank test. Cox proportional hazard regression will be performed to determine the independent associations between survival and potential explanatory variables.

Subgroup analyses will be performed. We will perform the analyses after excluding patients in whom liver cancer is diagnosed at their first MRI surveillance tests following the negative US prior to study enrollment. We will compare the tumor stages between patients diagnosed at the first tests and those diagnosed at later tests among patients undergoing noncontrast MRI surveillance. These analyses are intended to examine and exclude the possibility that the tumors already exist but are missed by the noncontrast US test prior to enrollment. In addition, we will examine the effects of the quality of the echogenic window to the liver and the 
parenchymal nodularity on our primary endpoints. Furthermore, we will explore the additional benefit of serum AFP testing in conjunction with noncontrast US or noncontrast MRI for the detection of early stage HCC. Two-sided $p$-values $<0.05$ are considered statistically significant. All statistical analyses will be performed using the SAS 9.2 software package.

\section{Monitoring}

Interventions used for this trial are US and MRI, which are the widely used imaging tests whose adverse events are reported very rarely [33]. Furthermore, in this trial, these imaging tests will be performed without the use of contrast media. Therefore, there is no harm particularly anticipated from this study other than the inherent minimal risks of the imaging tests themselves. Nevertheless, we will monitor and record any adverse events occurring after study enrolment at every visit. Any serious adverse event will be reported to our IRB. As this study pose a minimal risk to participants as stated above, we will nominate a physician independent of this study as an independent safety monitor (ISM), but there will not be a formal data and safety monitoring committee. The ISM will review annually the trial processes and accumulating data, and determine if the trial should be modified or discontinued.

\section{Discussion}

Our goal is to determine whether surveillance with noncontrast MRI can further improve the overall survival of patients at high risk of developing HCC, compared with surveillance using noncontrast US. To achieve this goal, we have designed this study as a two-arm randomized trial. However, no previous study has investigated the performance of noncontrast MRI in detecting HCC patients at early stages in a surveillance setting, although one study examined the diagnostic performance of noncontrast MRI to detect and diagnose HCC in a limited cohort of patients, primarily at very early or early stages [25]. Thus, we first set our primary endpoints as detection rate of early-stage HCC and false positive rate. At long-term follow-up of the current study cohort, we may observe a significant difference in overall survival between the two arms; if this is not possible, an appropriate sample size could be calculated based on the results of current trial for a future large-scale, multicenter trial.

We will perform surveillance every 6 months using both serum AFP and imaging tests, since the combined use of noncontrast US and AFP is recommended for surveillance in many practice guidelines published in Asia, including Korea [5, 6]. Inclusion of AFP may be a confounding factor in comparing the two imaging modalities. However, it would be unethical to exclude serum AFP testing from HCC surveillance for the sake of a clinical trial because the current evidence points to noncontrast US combined with serum AFP as the most effective surveillance strategy [34]. One advantage of this study design, however, is that this trial will allow us to assess the benefit of AFP performed in addition to US or MRI in HCC surveillance, by examining the proportion of cases where early-stage HCC is detected by AFP elevation with negative US or MRI results.

We will not designate experienced radiologists specifically for our surveillance US. Instead, as is currently practiced at our institution, well-trained hepatology fellows will perform surveillance US. In real-world clinical settings, many patients receive their surveillance US examinations in local community clinics instead of tertiary care centers, often by less-experienced US operators with less-than-optimal protocols or equipment [35]. It would be unrealistic to have all surveillance US examinations performed exclusively by a limited number of highly experienced operators.

MIRACLE-HCC is the first clinical trial to compare the effectiveness of noncontrast MRI and noncontrast US in the surveillance of HCC in at-risk patients. The results of this trial will show whether noncontrast MRI surveillance is superior to noncontrast US surveillance in the early detection of HCC, whether it results in fewer false referrals, and whether it can eventually improve the overall survival of HCC patients.

\section{Additional file}

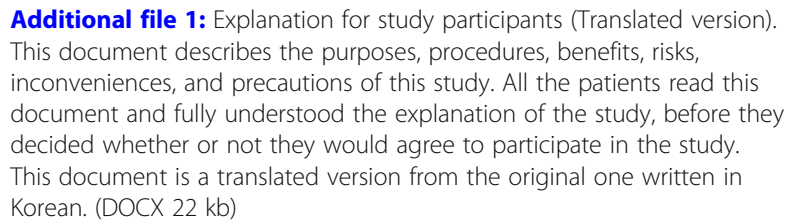

Additional file 1: Explanation for study participants (Translated version). This document describes the purposes, procedures, benefits, risks, inconveniences, and precautions of this study. All the patients read this document and fully understood the explanation of the study, before they decided whether or not they would agree to participate in the study. This document is a translated version from the original one written in Korean. (DOCX $22 \mathrm{~kb}$ )

\section{Abbreviations}

AFP: Alpha-fetoprotein; CT: Computed tomography; e-CASE: Electronic Case Reporting System with Accuracy, Safety, and Efficacy; GRE: Gradient-recalled echo; HBsAg: Hepatitis B surface antigen; HBV: Hepatitis B virus;

HCC: Hepatocellular carcinoma; HCV: Hepatitis C virus; IRB: Institutional review board; ISM: Independent safety monitor; LI-RADS: Liver Imaging Reporting and Data System; MIRACLE-HCC: Noncontrast magnetic resonance imaging versus noncontrast ultrasonography for surveillance of

hepatocellular carcinoma; MRI: Magnetic resonance imaging; NEX: Number of excitations; US: Ultrasonography

\section{Acknowledgements}

The authors would like to thank Yoo Ri Im, BS, (research nurse, Severance Hospital, Yonsei University College of Medicine, Seoul, Korea) for assisting with patient enrollment and data management.

\section{Funding}

This study has received funding by the National R\&D Program for Cancer Control, Ministry of Health \& Welfare, Korea (Grant \#1520160). The funder has no role in study design, data collection and analysis, decision to publish, or preparation of the manuscript. 


\section{Availability of data and materials}

Patient enrollment was finished on November 29, 2017, and follow-up to see primary and secondary endpoints is still on-going. Data sharing is not applicable to this article as no datasets were generated or analyzed during the current study.

\section{Authors' contributions}

MJK and DYK conceptualized this study. All six authors (CA, DYK, JYC, KHH, YHR, and MJK) participated in study design. DYK and KHH played major roles in patient enrollment. YHR provided professional statistical assistance. DYK, $\mathrm{KHH}, J Y C$, and MJK established the protocols for surveillance US and MRI. MJK, JYC, and CA performed the interpretation of surveillance MRI. CA was a major contributor in writing the manuscript. All authors read and approved the final manuscript.

\section{Ethics approval and consent to participate}

This study has been approved by our institutional review board (Severance Hospital, Yonsei University College of Medicine; e-IRB: http://eirb.yuhs.ac; IRB number: 4-2015-0029). Any modifications to the protocol will be implemented only after they are approved by the IRB and notified to all participating investigators and patients. We obtained informed written consent to participate in this study from all participating patients. The translated version of the model informed consent form is provided as an additional document (see Additional file 1).

\section{Consent for publication}

Not applicable.

\section{Competing interests}

The authors declare that they have no competing interests.

\section{Publisher's Note}

Springer Nature remains neutral with regard to jurisdictional claims in published maps and institutional affiliations.

\section{Author details}

'Department of Radiology, Research Institute of Radiological Science, Yonsei University College of Medicine, 50 Yonsei-ro, Seodaemun-gu, Seoul 03722, South Korea. ${ }^{2}$ Department of Internal Medicine, Yonsei University College of Medicine, Seoul, South Korea. ${ }^{3}$ Biostatistics Collaboration Unit, Medical Research Center, Yonsei University College of Medicine, Seoul, South Korea.

Received: 12 December 2017 Accepted: 17 September 2018 Published online: 24 September 2018

\section{References}

1. Zhang BH, Yang BH, Tang ZY. Randomized controlled trial of screening for hepatocellular carcinoma. J Cancer Res Clin Oncol. 2004:130(7):417-22.

2. van Meer S, de Man RA, Coenraad MJ, Sprengers D, van Nieuwkerk KM, Klumpen $\mathrm{HJ}$, et al. Surveillance for hepatocellular carcinoma is associated with increased survival: results from a large cohort in the Netherlands. J Hepatol. 2015;63(5):1156-63.

3. Singal AG, Pillai A, Tiro J. Early detection, curative treatment, and survival rates for hepatocellular carcinoma surveillance in patients with cirrhosis: a meta-analysis. PLoS Med. 2014;11:e1001624.

4. Mittal S, Kanwal F, Ying J, Chung R, Sada YH, Temple S, et al. Effectiveness of surveillance for hepatocellular carcinoma in clinical practice: a United States cohort. J Hepatol. 2016;65(6):1148-54.

5. Omata M, Cheng AL, Kokudo N, Kudo M, Lee JM, Jia J, et al. Asia-Pacific clinical practice guidelines on the management of hepatocellular carcinoma: a 2017 update. Hepatol Int. 2017;11(4):317-70.

6. Lee JM, Park JW, Choi BI. 2014 KLCSG-NCC Korea practice guidelines for the management of hepatocellular carcinoma: HCC diagnostic algorithm. Dig Dis. 2014;32(6):764-77.

7. Heimbach JK, Kulik LM, Finn RS, Sirlin CB, Abecassis MM, Roberts LR, et al. AASLD guidelines for the treatment of hepatocellular carcinoma. Hepatology. 2018;67(1):358-80.

8. European Association For The Study Of The Liver, European Organisation For Research And Treatment Of Cancer. EASL-EORTC clinical practice guidelines: management of hepatocellular carcinoma. J Hepatol. 2012;56(4):908-43.
9. Kim KA, Kim MJ, Choi JY, Chung YE. Development of hepatocellular carcinomas in patients with absence of tumors on a prior ultrasound examination. Eur J Radiol. 2012;81(7):1450-4.

10. Singal A, Volk ML, Waljee A, Salgia R, Higgins P, Rogers MA, et al. Metaanalysis: surveillance with ultrasound for early-stage hepatocellular carcinoma in patients with cirrhosis. Aliment Pharmacol Ther. 2009;30(1):37-47.

11. Singal AG, Conjeevaram HS, Volk ML, Fu S, Fontana RJ, Askari F, et al. Effectiveness of hepatocellular carcinoma surveillance in patients with cirrhosis. Cancer Epidemiol Biomark Prev. 2012;21(5):793-9.

12. Chou R, Cuevas C, Fu R, Devine B, Wasson N, Ginsburg A, et al. Imaging techniques for the diagnosis of hepatocellular carcinoma: a systematic review and meta-analysis. Ann Intern Med. 2015;162(10):697-711.

13. Sinn DH, Yi J, Choi MS, Choi D, Gwak GY, Paik YH, et al. Incidence and risk factors for surveillance failure in patients with regular hepatocellular carcinoma surveillance. Hepatol Int. 2013;7(4):1010-8.

14. Singal AG, Nehra M, Adams-Huet B, Yopp AC, Tiro JA, Marrero JA, et al. Detection of hepatocellular carcinoma at advanced stages among patients in the HALT-C trial: where did surveillance fail? Am J Gastroenterol. 2013;108(3):425-32.

15. Del Poggio P, Olmi S, Ciccarese F, Di Marco M, Rapaccini GL, Benvegnu L, et al. Factors that affect efficacy of ultrasound surveillance for early stage hepatocellular carcinoma in patients with cirrhosis. Clin Gastroenterol Hepatol. 2014;12(11):1927-33.e2.

16. Besa C, Lewis S, Pandharipande PV, Chhatwal J, Kamath A, Cooper N, et al. Hepatocellular carcinoma detection: diagnostic performance of a simulated abbreviated MRI protocol combining diffusion-weighted and T1-weighted imaging at the delayed phase post gadoxetic acid. Abdom Radiol (NY). 2017:42(1):179-90.

17. Kim SY, An J, Lim YS, Han S, Lee JY, Byun JH, et al. MRI with liver-specific contrast for surveillance of patients with cirrhosis at high risk of hepatocellular carcinoma. JAMA Oncol. 2017:3(4):456-63.

18. Marks RM, Ryan A, Heba ER, Tang A, Wolfson TJ, Gamst AC, et al. Diagnostic per-patient accuracy of an abbreviated hepatobiliary phase gadoxetic acid-enhanced MRI for hepatocellular carcinoma surveillance. AJR Am J Roentgenol. 2015:204(3):527-35.

19. Park JH, Park MS, Lee SJ, Jeong WK, Lee JY, Park MJ, et al. Contrastenhanced US with Perfluorobutane(Sonazoid) used as a surveillance test for hepatocellular carcinoma (HCC) in cirrhosis (SCAN): an exploratory crosssectional study for a diagnostic trial. BMC Cancer. 2017;17(1):279.

20. Pocha C, Dieperink E, McMaken KA, Knott A, Thuras P, Ho SB. Surveillance for hepatocellular cancer with ultrasonography vs. computed tomography a randomised study. Aliment Pharmacol Ther. 2013;38(3):303-12.

21. Chung YE, Kim MJ, Park MS, Choi JY, Lim JS, Kim KA, et al. The impact of CT follow-up interval on stages of hepatocellular carcinomas detected during the surveillance of patients with liver cirrhosis. AJR Am J Roentgenol. 2012:199(4):816-21.

22. Brenner DJ, Hall EJ. Computed tomography--an increasing source of radiation exposure. N Engl J Med. 2007:357(22):2277-84.

23. Yu NC, Chaudhari V, Raman SS, Lassman C, Tong MJ, Busuttil RW, et al. CT and MRI improve detection of hepatocellular carcinoma, compared with ultrasound alone, in patients with cirrhosis. Clin Gastroenterol Hepatol. 2011:9(2):161-7.

24. Tedeschi E, Caranci F, Giordano F, Angelini V, Cocozza S, Brunetti A. Gadolinium retention in the body: what we know and what we can do. Radiol Med. 2017;122(8):589-600.

25. Kim YK, Kim YK, Park HJ, Park MJ, Lee WJ, Choi D. Noncontrast MRI with diffusion-weighted imaging as the sole imaging modality for detecting liver malignancy in patients with high risk for hepatocellular carcinoma. Magn Reson Imaging. 2014;32(6):610-8.

26. Oh CM, Won YJ, Jung KW, Kong HJ, Cho H, Lee JK, et al. Cancer statistics in Korea: incidence, mortality, survival, and prevalence in 2013. Cancer Res Treat. 2016;48(2):436-50.

27. Goh GB, Chang PE, Tan CK. Changing epidemiology of hepatocellular carcinoma in Asia. Best Pract Res Clin Gastroenterol. 2015:29(6):919-28.

28. Lee KS, Chang HS, Lee SM, Park EC. Economic burden of Cancer in Korea during 2000-2010. Cancer Res Treat. 2015:47(3):387-98.

29. Jung KW, Won YJ, Oh CM, Kong HJ, Lee DH, Lee KH. Cancer statistics in Korea: incidence, mortality, survival, and prevalence in 2014. Cancer Res Treat. 2017;49(2):292-305. 
30. American College of Radiology: Liver imaging reporting and data system. 2017. http://www.acr.org/Quality-Safety/Resources/LIRADS.

Accessed 2 Nov 2017.

31. Korean Society of Ultrasound in Medicine: Practice guidelines for the performance of ultrasound examinations. 2014. http://www.ultrasound.or.kr. Accessed 2 July 2017.

32. Machin D, Campbell MJ, Tan SB, Tan SH. Sample size tables for clinical studies. 3rd ed. Chichester: United Kingdom: Wiley-Blackwell; 2008.

33. The United States Food and Drug Administration. http://www.fda.gov (2017). Accessed 6 Mar 2018.

34. Song PP, Xia JF, Inagaki Y, Hasegawa K, Sakamoto Y, Kokudo N, et al. Controversies regarding and perspectives on clinical utility of biomarkers in hepatocellular carcinoma. World J Gastroenterol. 2016;22(1):262-74.

35. Choi Jl, Jung SE, Kim PN, Cha SH, Jun JK, Lee HY, et al. Quality assurance in ultrasound screening for hepatocellular carcinoma using a standardized phantom and standard clinical images: a 3-year national investigation in Korea. J Ultrasound Med. 2014;33(6):985-95.

Ready to submit your research? Choose BMC and benefit from:

- fast, convenient online submission

- thorough peer review by experienced researchers in your field

- rapid publication on acceptance

- support for research data, including large and complex data types

- gold Open Access which fosters wider collaboration and increased citations

- maximum visibility for your research: over $100 \mathrm{M}$ website views per year

At $\mathrm{BMC}$, research is always in progress.

Learn more biomedcentral.com/submissions 